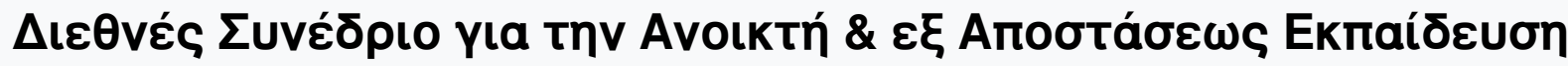

Tóp. 7, Ap. 1A (2013)

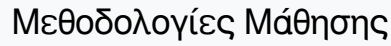

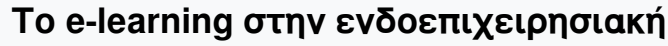

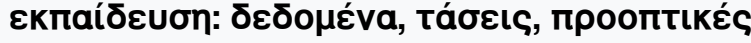

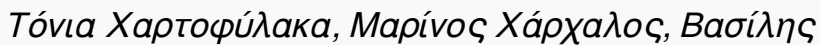

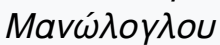

doi: $10.12681 /$ icodl. 547 


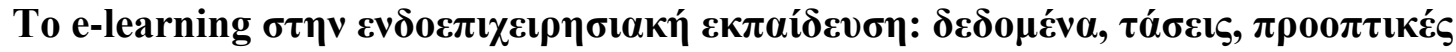

\section{Workplace e-learning: data, trends and perspectives}

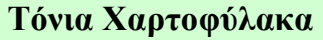 \\ Instructional Designer \\ DigiMagix \\ $\Delta \rho . A v \theta \rho \omega \pi \imath \tau \tau \iota \kappa \omega ́ v \Sigma \pi 0 v \delta \omega ́ v$ \\ thartofylaka@digimagix.gr
}

\author{
Mapívos Xó $\chi \alpha \lambda$ os \\ Software Engineer, DigiMagix \\ MSc in e-learning \\ mcharchalos@digimagix.gr
}

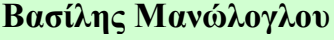 \\ CEO, DigiMagix \\ Software Engineer \\ vmanol@digimagix.gr
}

\begin{abstract}
The present paper underlines the necessity to designate the results of the e-learning programs that are being realized in workplace learning in our country, in order to start a fruitful dialogue regarding the perspectives of the specific learning methodology. The paper begins with a brief bibliographic survey in the field of workplace learning; it continues with the presentation of the general status of elearning in industries globally as well as the particularities in our country. It concludes by setting the concern regarding the lack of enough data that blocks the right view of the trends and perspectives that workplace e-learning may acquire in our country.
\end{abstract}

\section{Пврі́ $\eta \psi \eta$}

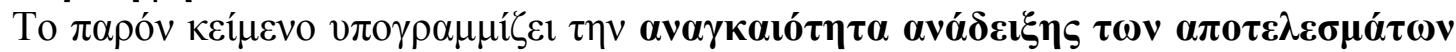

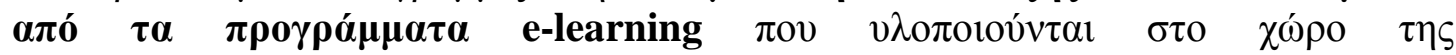

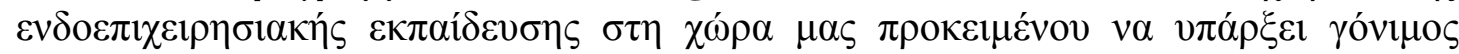

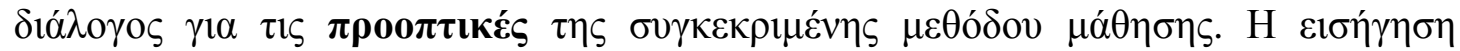

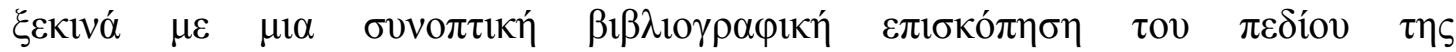

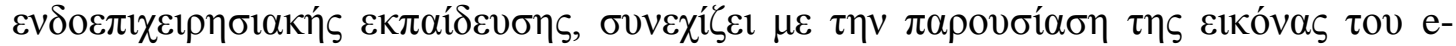

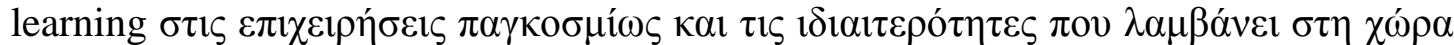

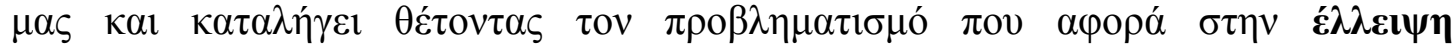

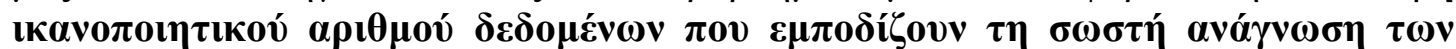

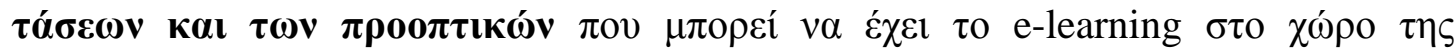

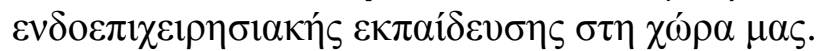

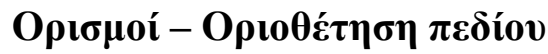

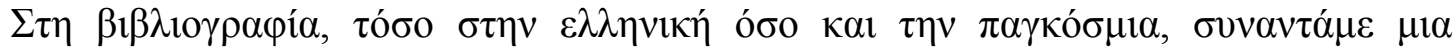

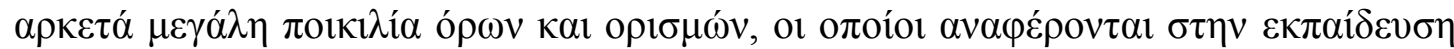

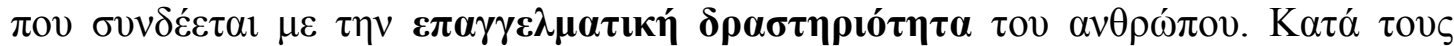

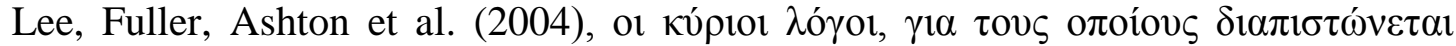

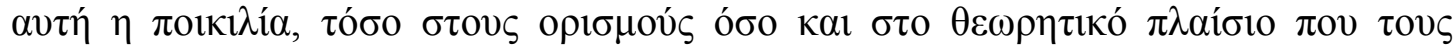

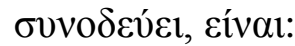

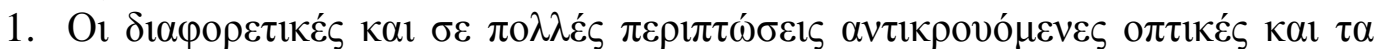

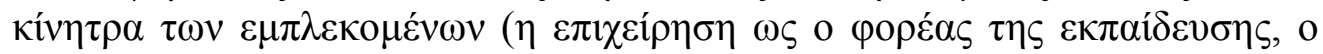

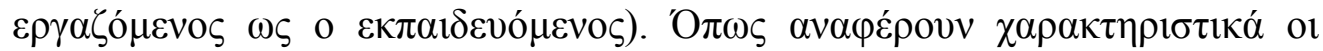

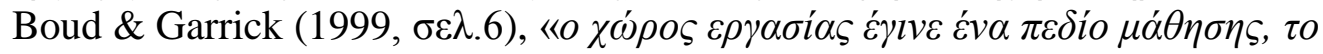

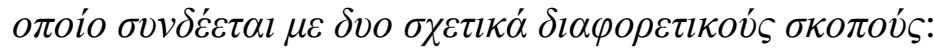




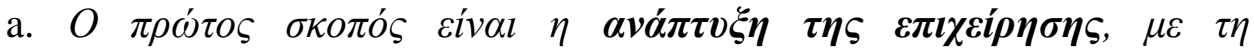

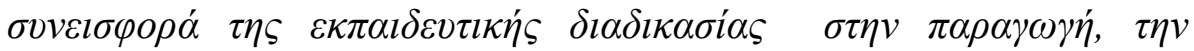

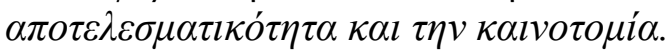

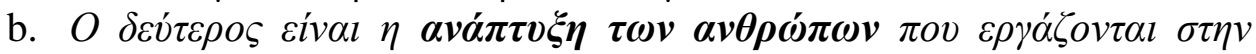

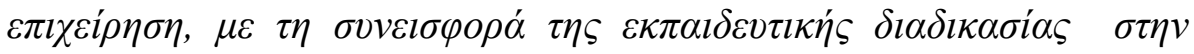

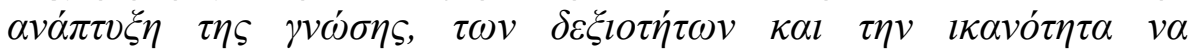

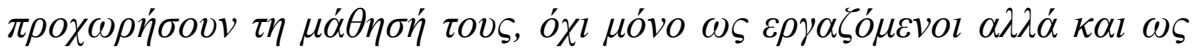

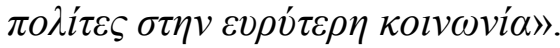

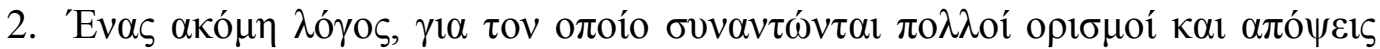

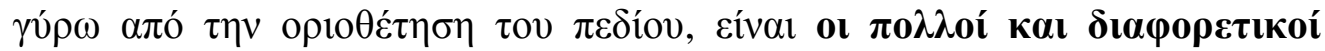

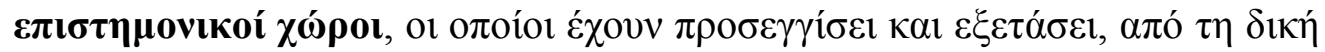

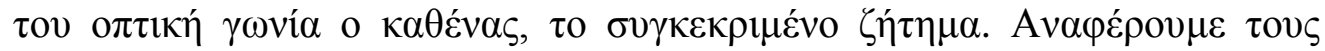

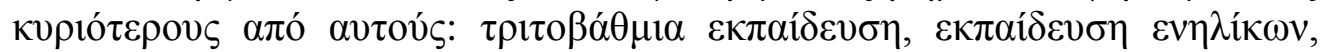
$\varepsilon \pi \alpha \gamma \gamma \varepsilon \lambda \mu \alpha \tau i \kappa \eta ́ ~ \varepsilon \kappa \pi \alpha i ́ \delta \varepsilon v \sigma \eta, \delta 1 o i ́ \kappa \eta \sigma \eta ~ \varepsilon \pi \imath \chi \varepsilon i \rho \eta ́ \sigma \varepsilon \omega v, ~ \delta 1 \alpha \chi \varepsilon i ́ p 1 \sigma \eta ~ \alpha v \theta \rho \omega \pi i ́ v \omega v$

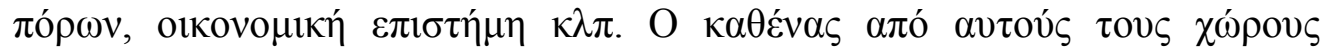

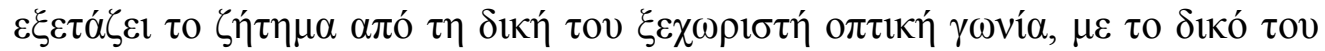

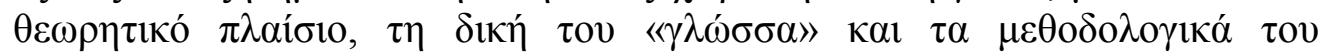
$\varepsilon \rho \gamma \alpha \lambda \varepsilon i ́ \alpha$.

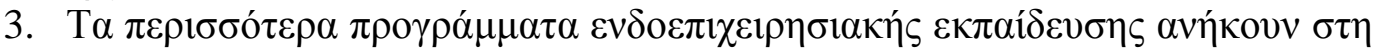

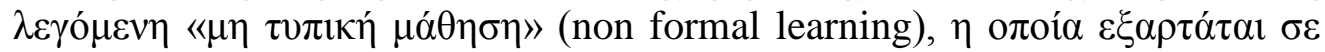

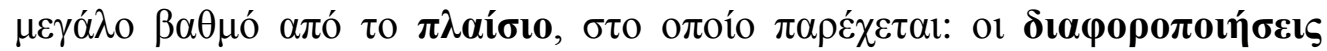

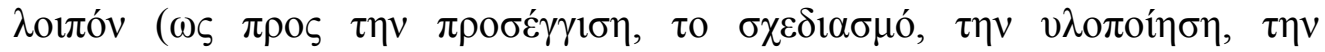

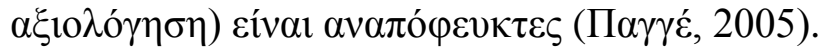

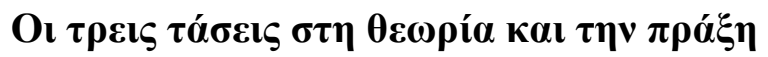

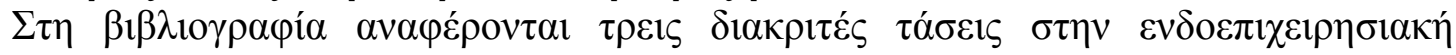

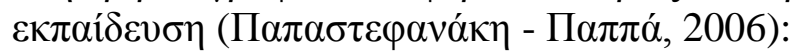

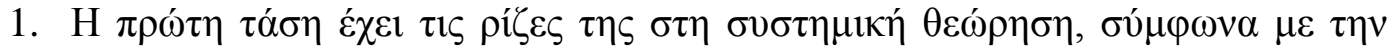

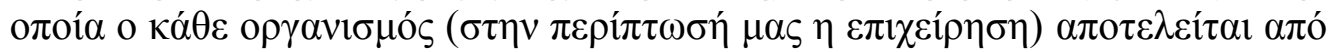

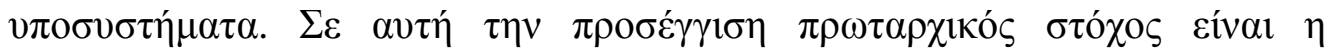

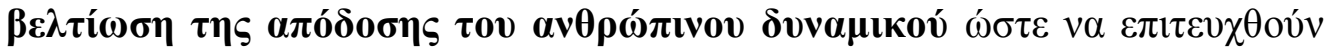

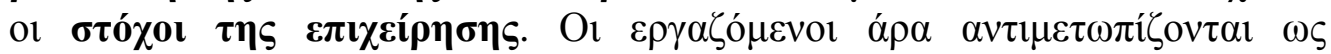

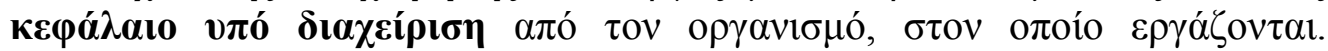

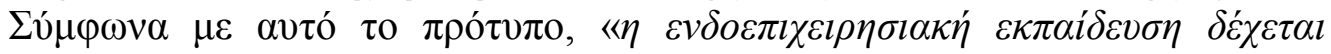

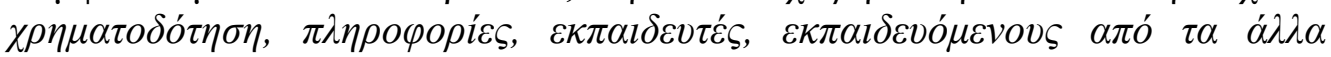

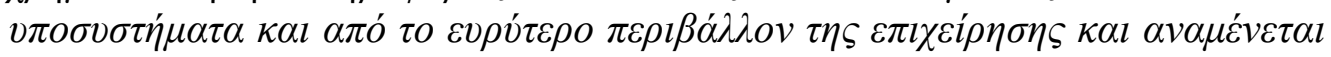

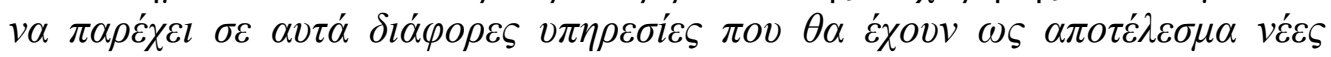
$\gamma v \omega ́ \sigma \varepsilon l \varsigma, \beta \varepsilon \lambda \tau \imath \omega \mu \varepsilon \dot{v \varepsilon \varepsilon \varsigma ~ \iota \kappa \alpha v o ́ \tau \eta \tau \varepsilon \varsigma ~ \kappa \alpha l ~ \theta \varepsilon \tau \iota \kappa o ́ \tau \varepsilon \rho \varepsilon \varsigma ~ \sigma \tau \alpha ́ \sigma \varepsilon ı \varsigma » ~(П \alpha \pi \alpha \sigma \tau \varepsilon \varphi \alpha v \alpha ́ \kappa \eta ~-~}$

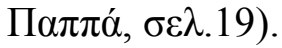

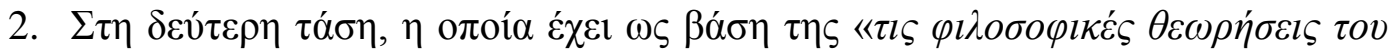

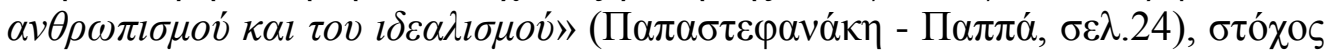

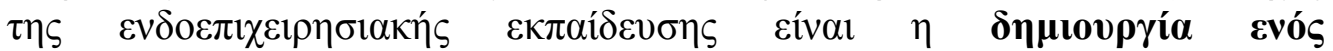

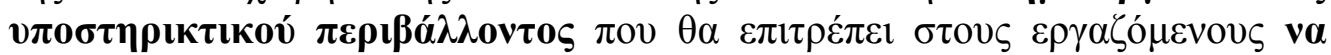

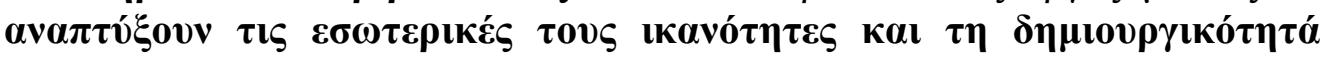

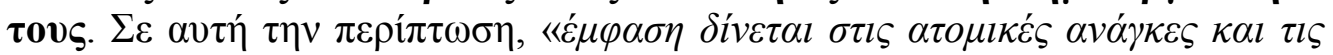

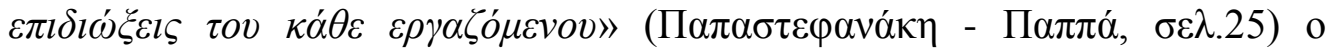

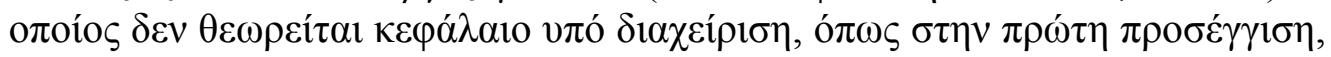

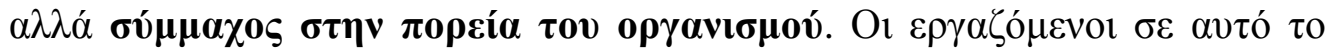

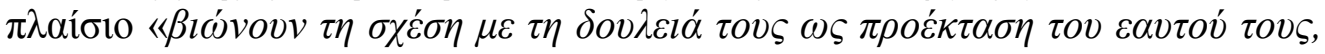

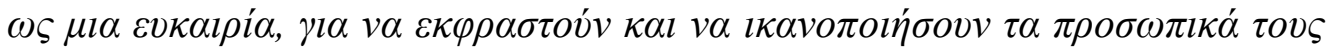




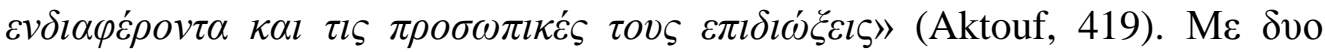

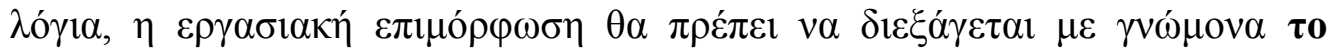

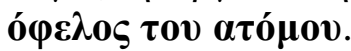

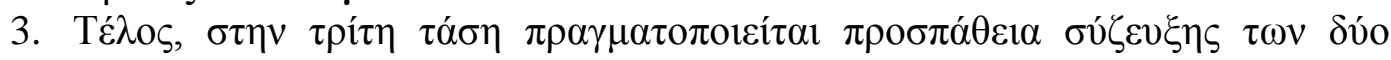

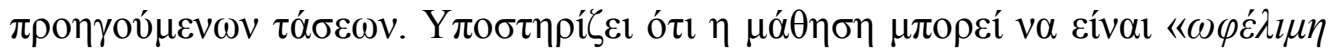

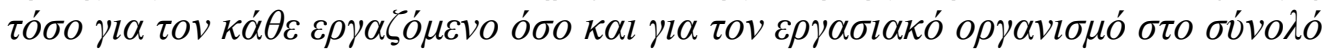

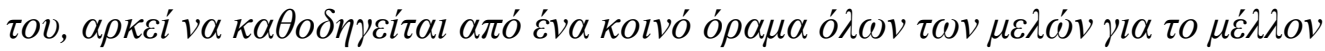

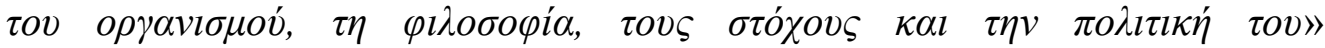

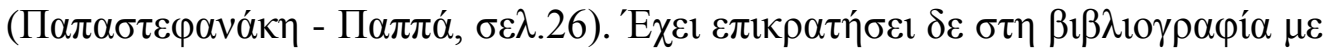

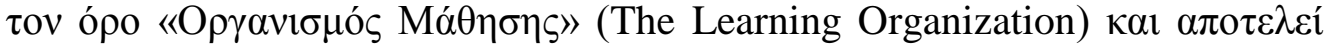

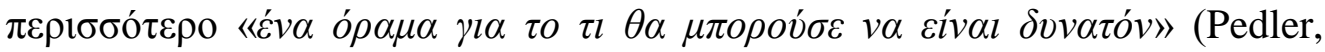
Burgoyne \& Boydell, $\sigma \varepsilon \lambda .3)$.

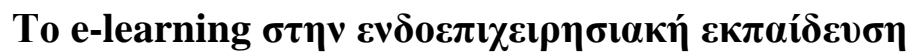

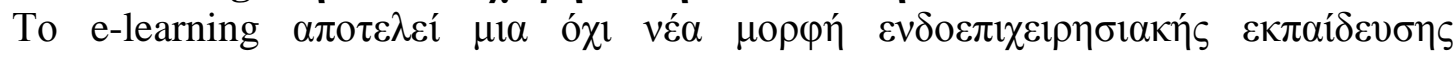

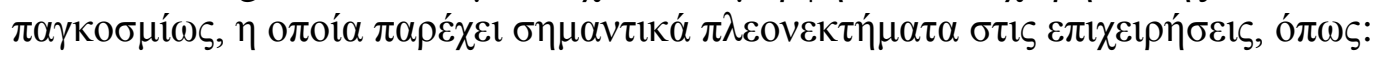

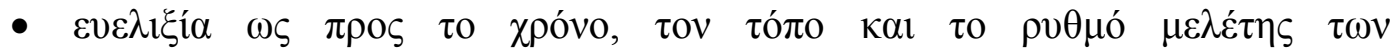

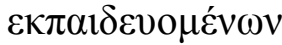

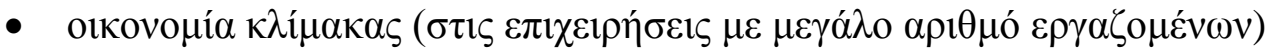

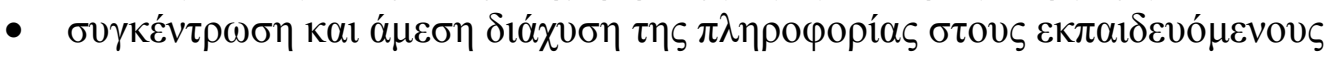

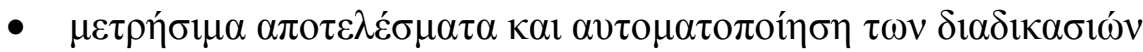

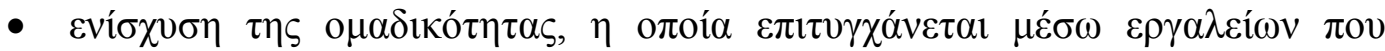

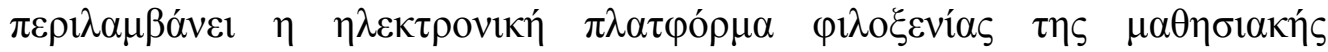
$\delta i \alpha \delta 1 \kappa \alpha \sigma i \alpha \varsigma$

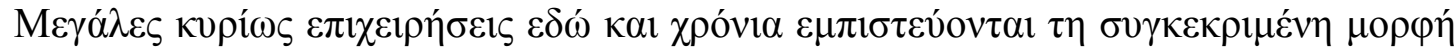

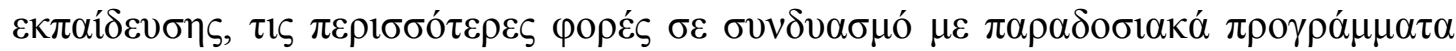

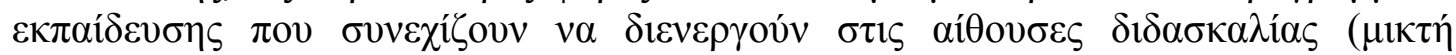

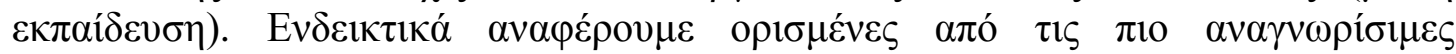
$\varepsilon \pi \imath \chi \varepsilon \imath \eta_{\sigma \varepsilon \varepsilon \iota:}$

- H

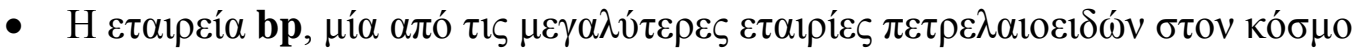

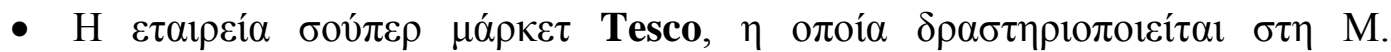

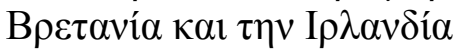

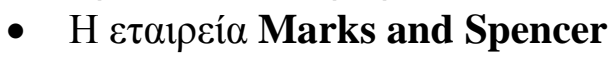

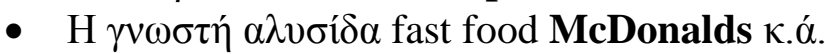

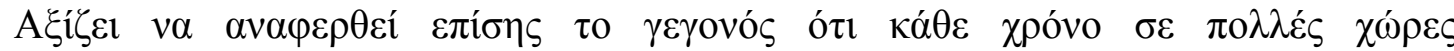

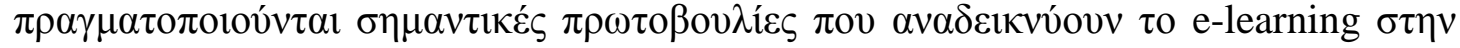

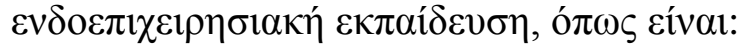

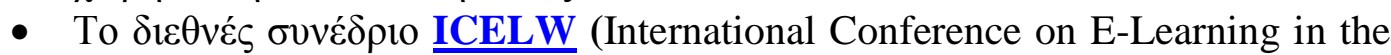

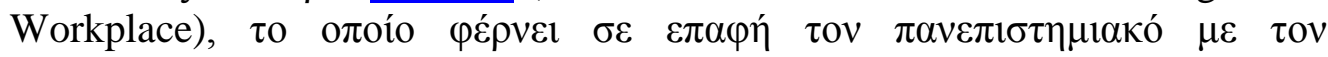

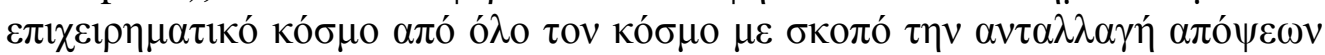

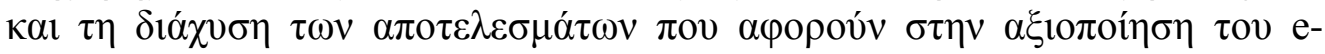

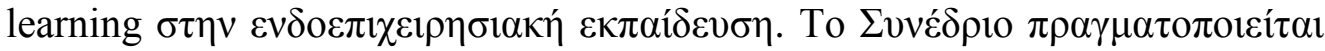

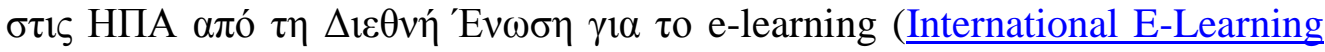
Association).

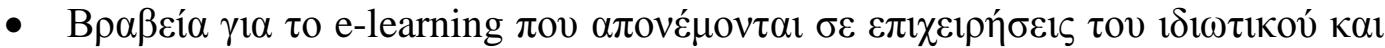

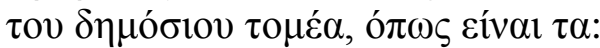

$\circ$ e-learning awards 
- International E-Learning Awards, ó $\pi 0 v \beta \rho \alpha \beta \varepsilon v ́ o v \tau \alpha$ $\tau \alpha \kappa \alpha \lambda v ́ \tau \varepsilon \rho \alpha$

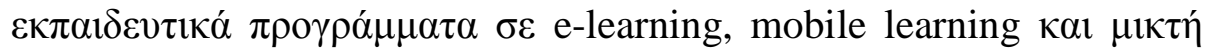

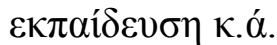

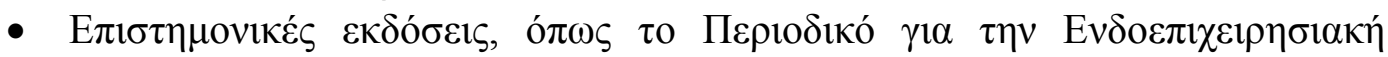

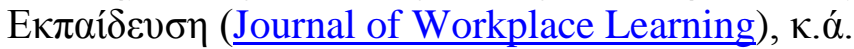

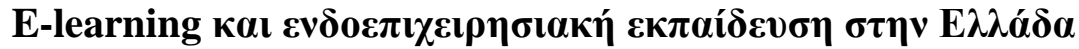

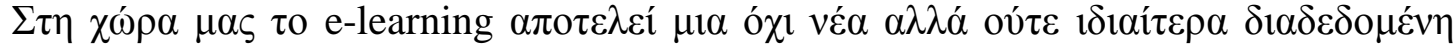

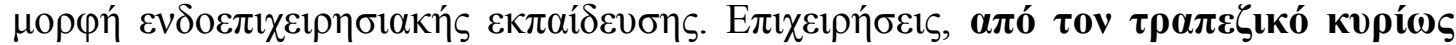

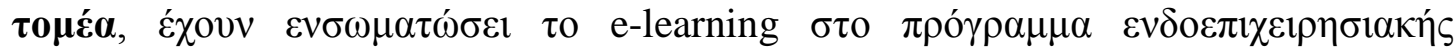

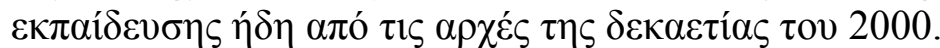

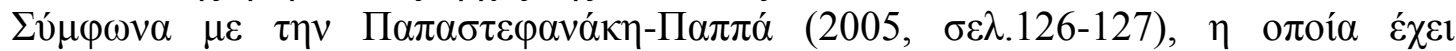

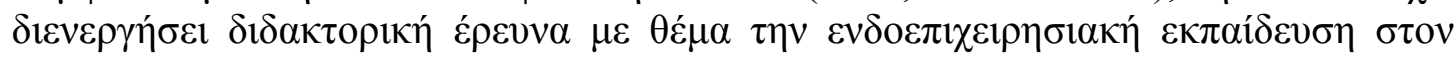

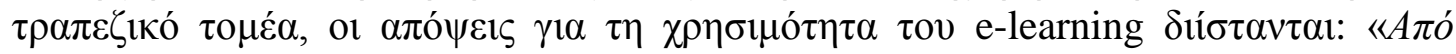

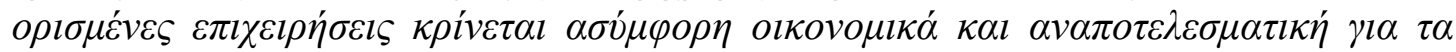

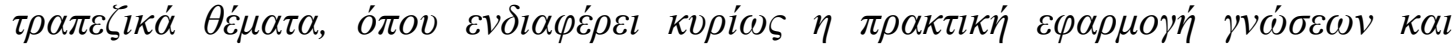

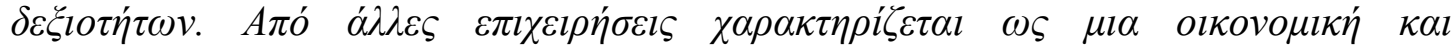

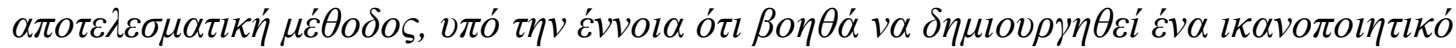

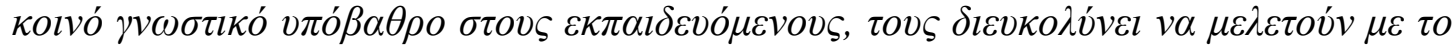

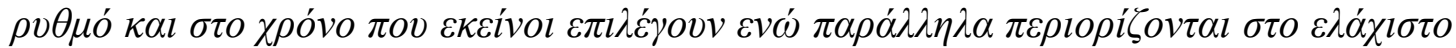

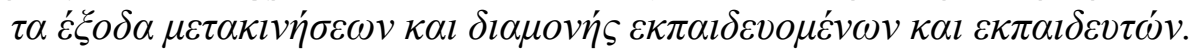

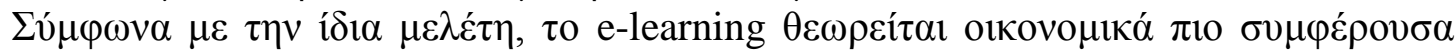

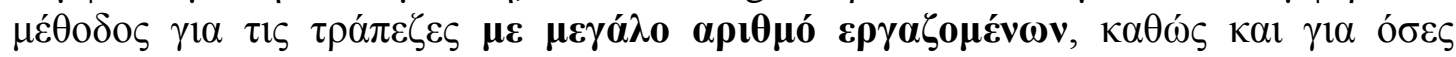

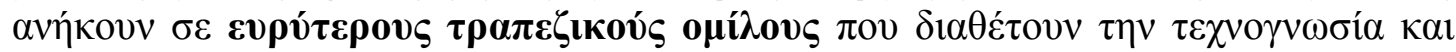

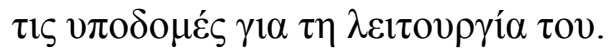

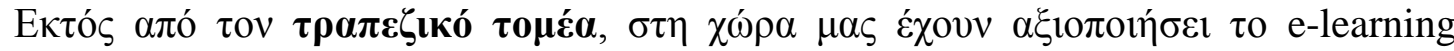

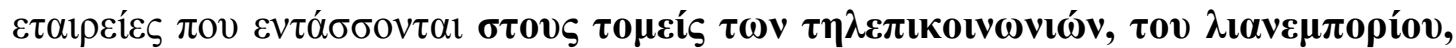

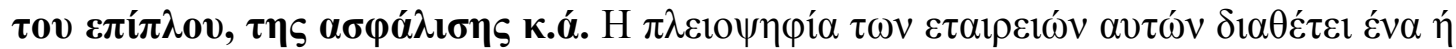

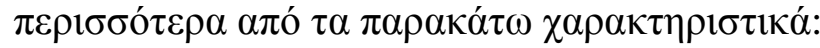

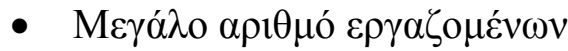

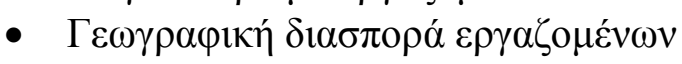

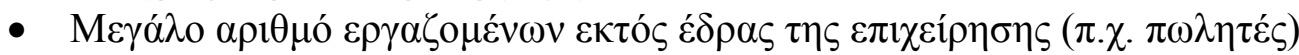

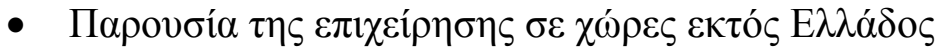

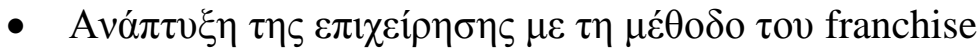

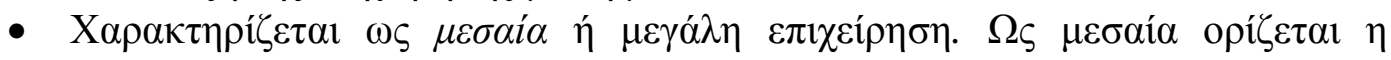

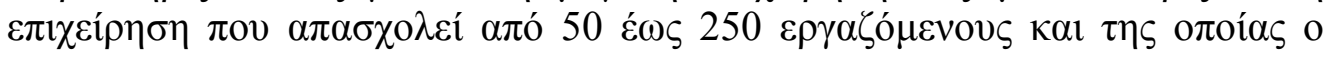

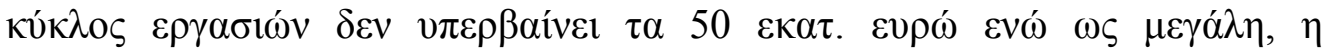

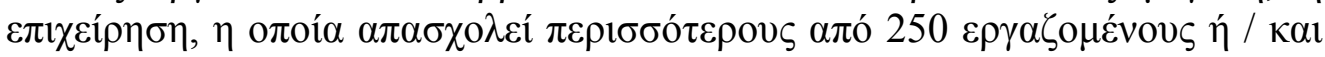

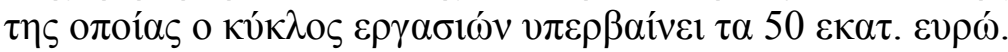

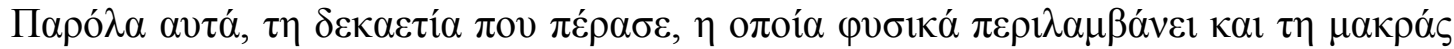

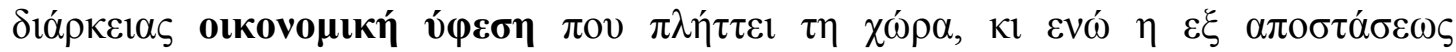

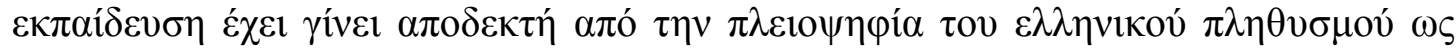

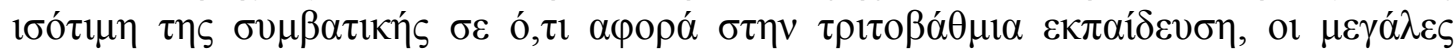

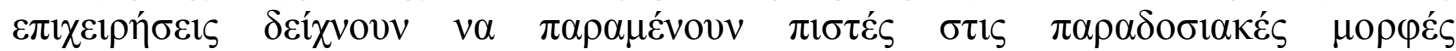

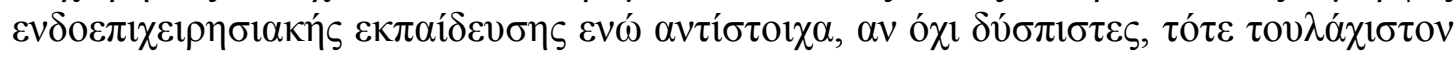

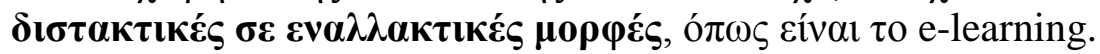

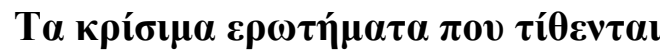

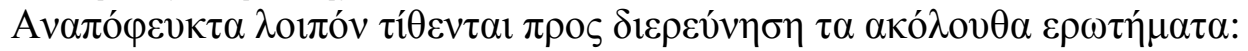




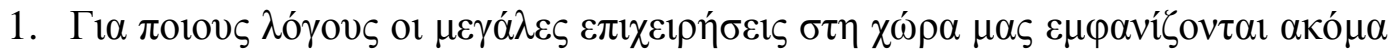

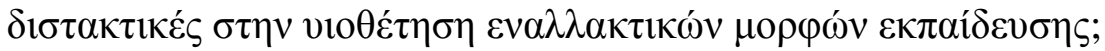

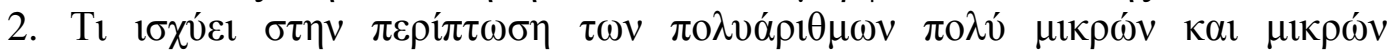

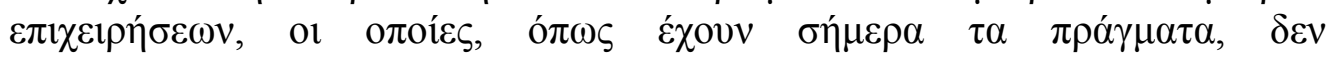

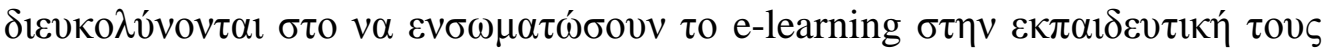

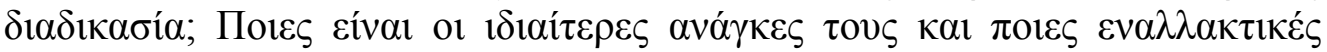
$\lambda$

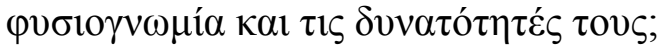

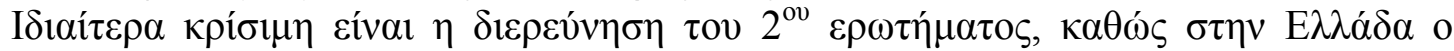

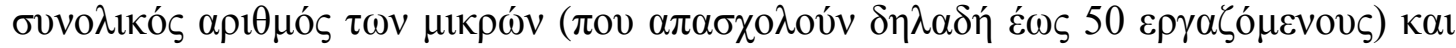

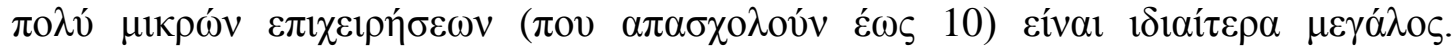

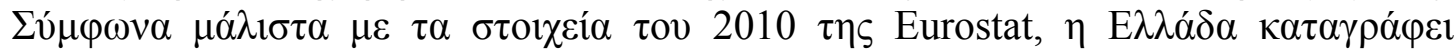

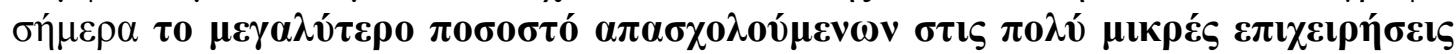

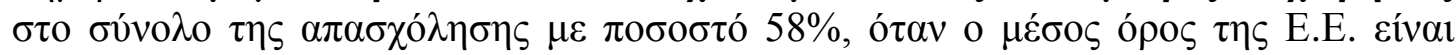

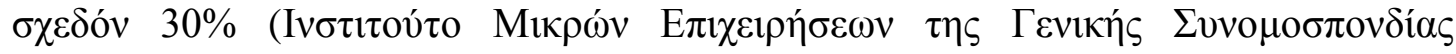

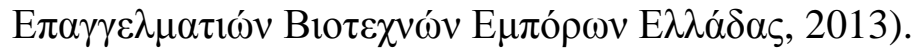

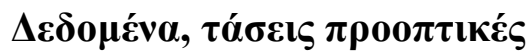

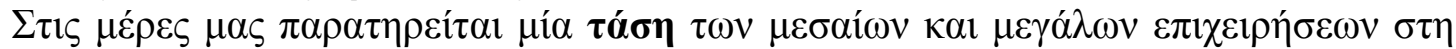

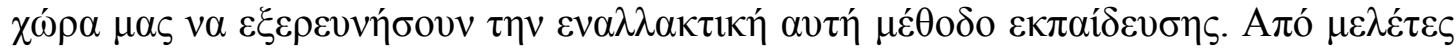

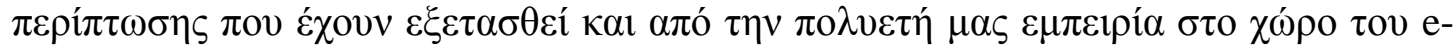

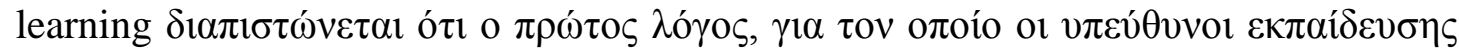

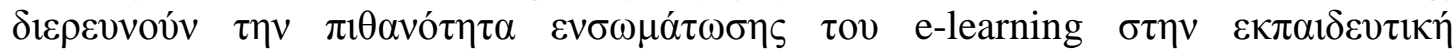

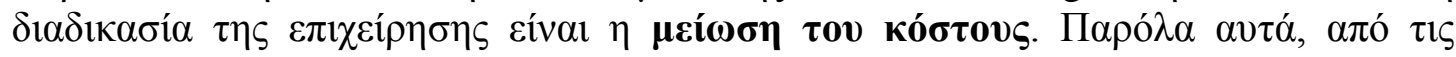

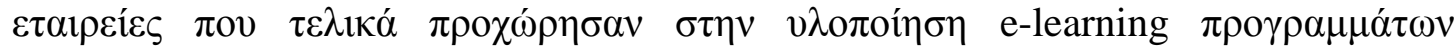

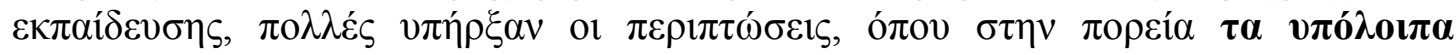

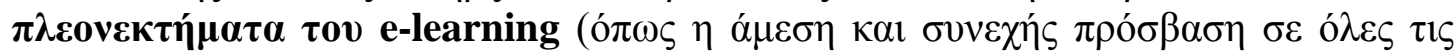

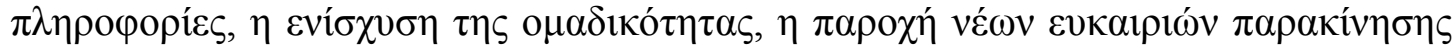

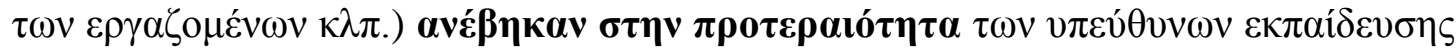

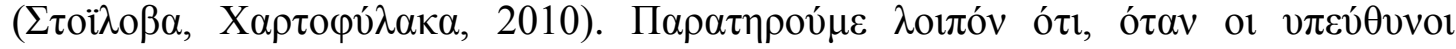

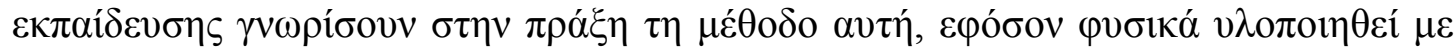

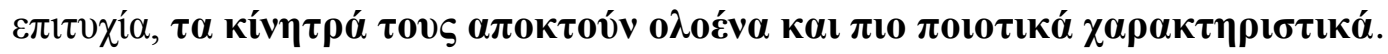

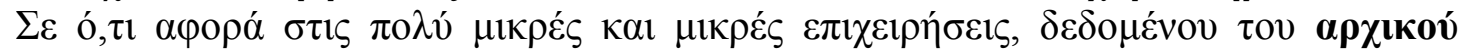

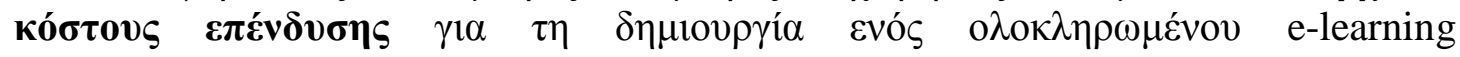

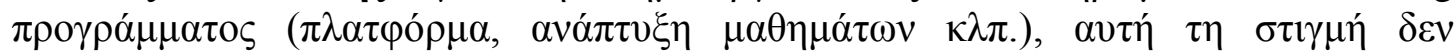

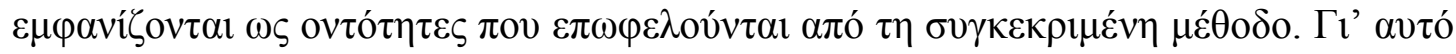

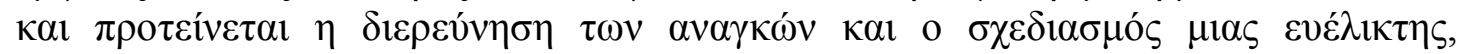

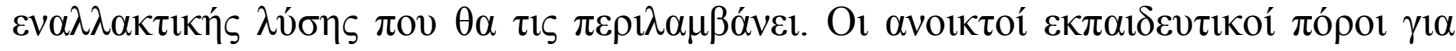

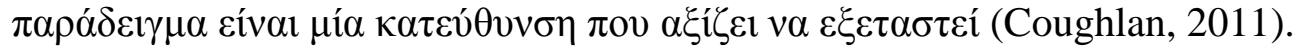

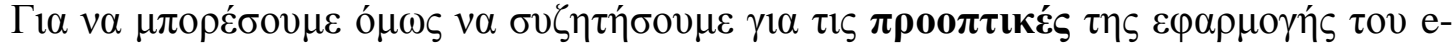

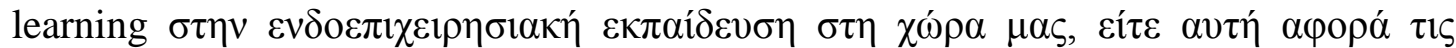

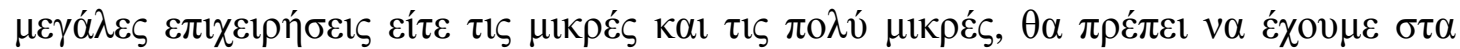

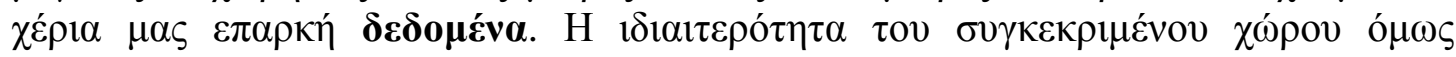

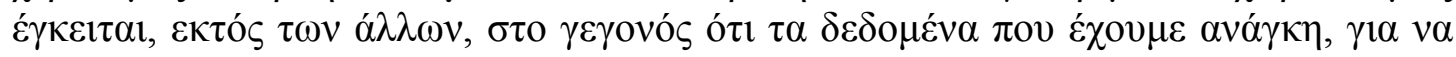

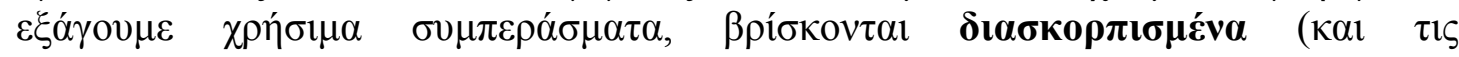

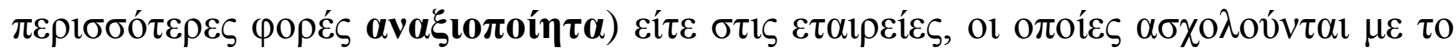

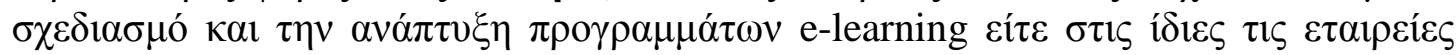

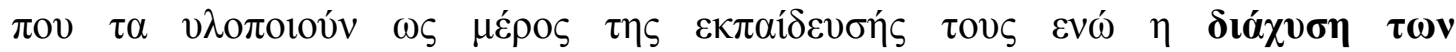

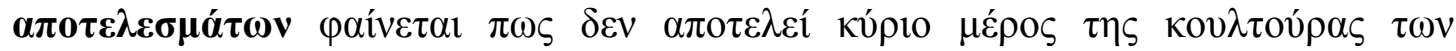
$\varepsilon \lambda \lambda \eta v i \kappa \omega ́ v ~ \varepsilon \pi \imath \chi \varepsilon \imath \rho \eta ́ \sigma \varepsilon \omega \nu$. 


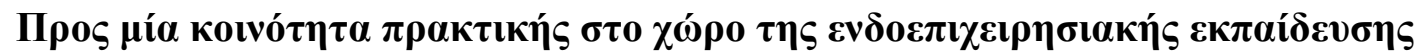

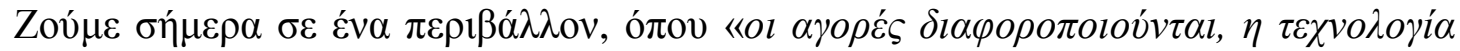

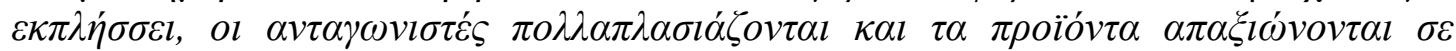

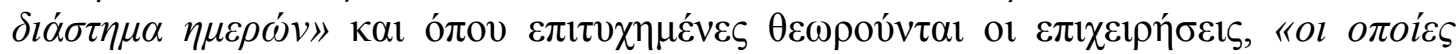

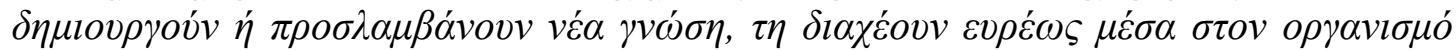

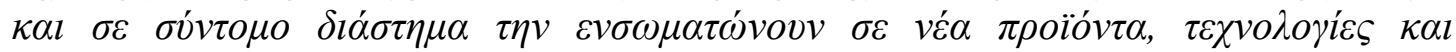

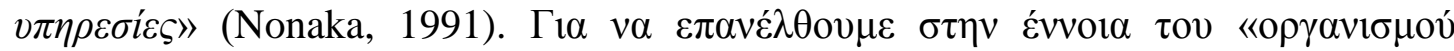

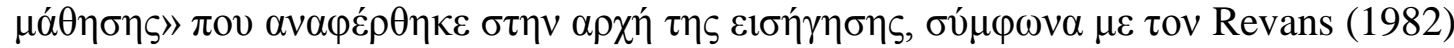

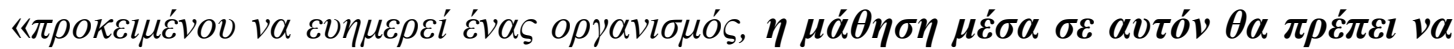

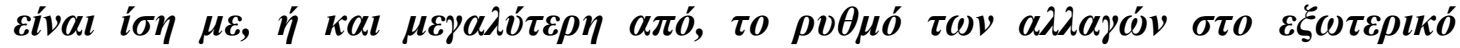

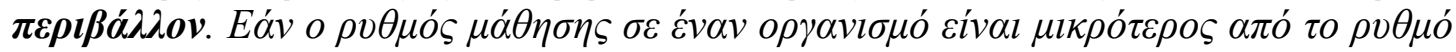

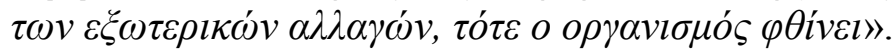

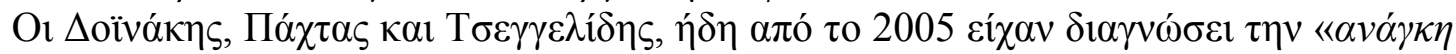

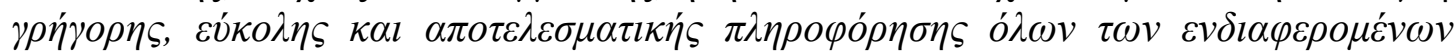

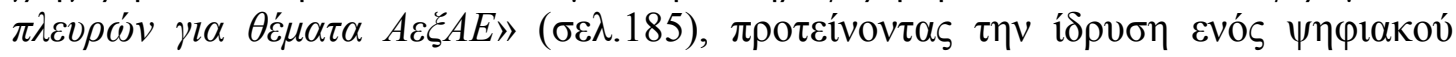

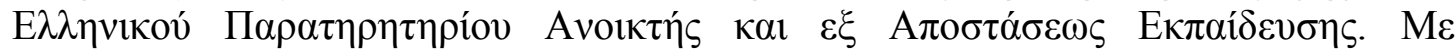

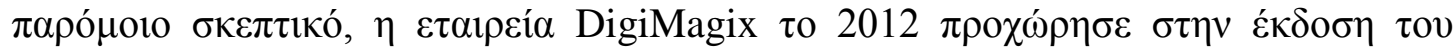

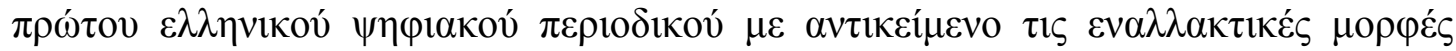

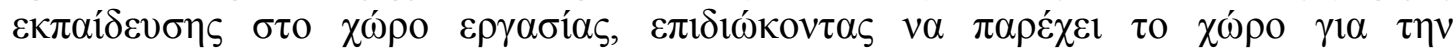

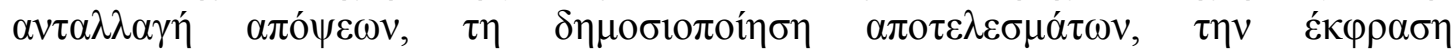

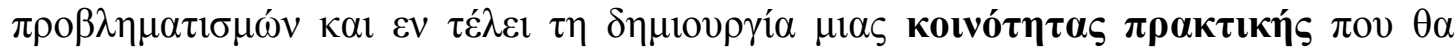

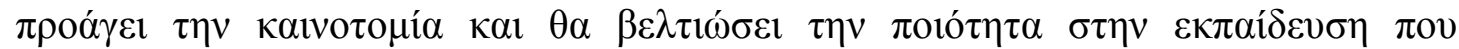

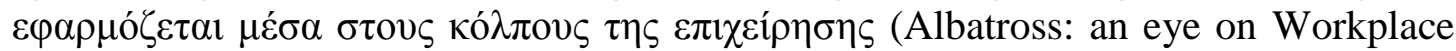

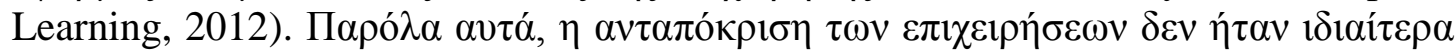

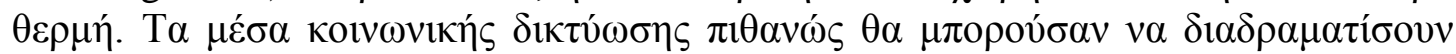

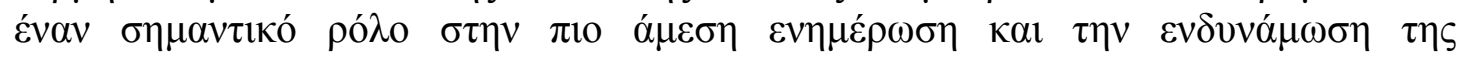

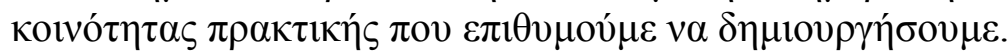

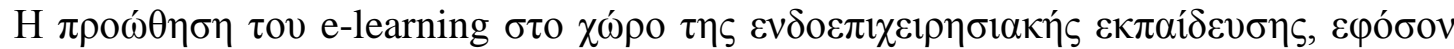

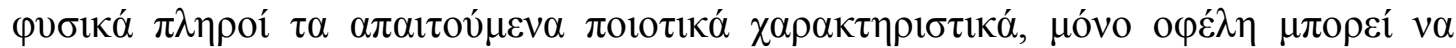

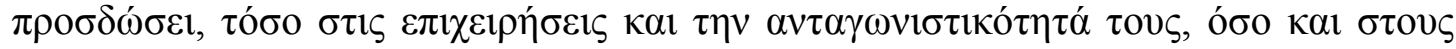

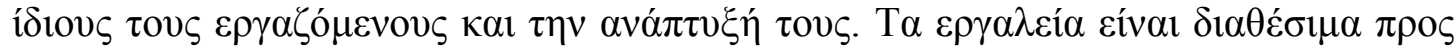

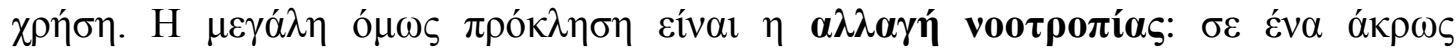

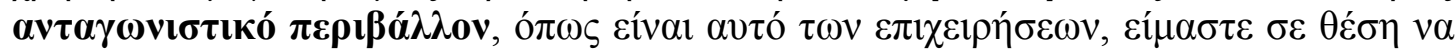

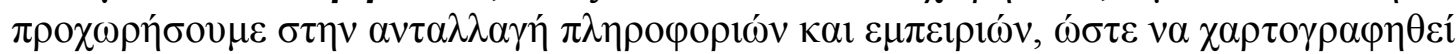

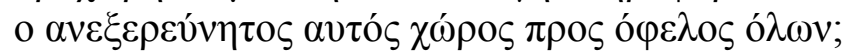

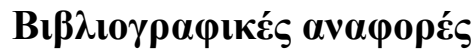

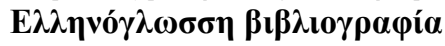

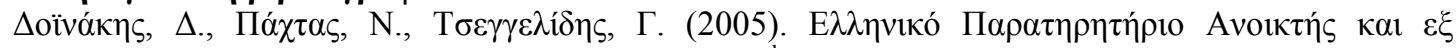

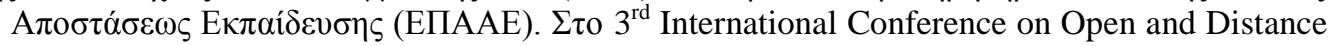

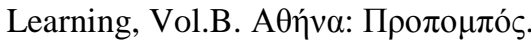

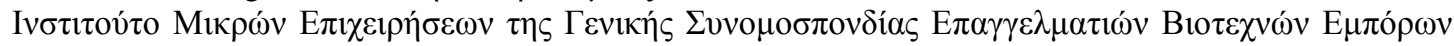

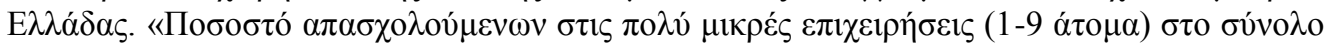

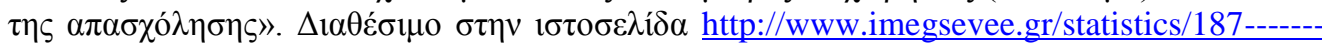

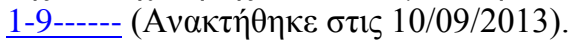

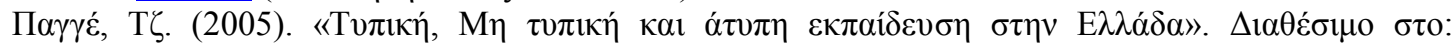
http://www.google.gr/url?sa=t\&rct=j\&q=\&esrc=s\&source=web\&cd=9\&ved=0CE0QFjAI\&ur l=http\%3A\%2F\%2Fequipe.up.pt\%2FRESOURCES\%2FCasestudies\%2Foriginal languages\% 2FIoannina_GR.doc\&ei=mFt6Ut7IGeSK7AaQv4D4Dw\&usg=AFQjCNHFVvaowlsXEIpVm

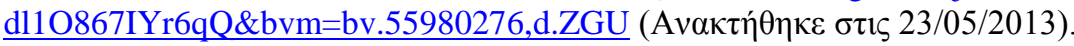




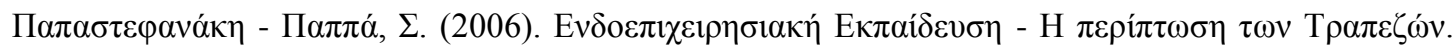

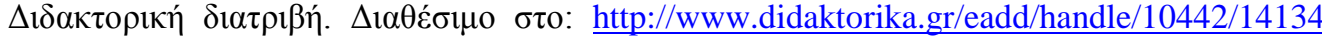

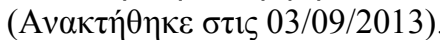

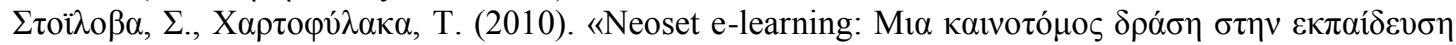

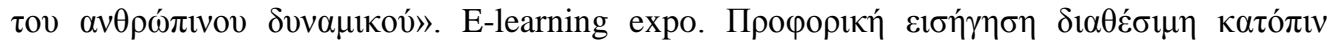

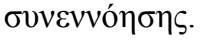

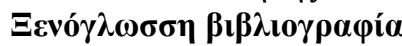

Aktouf O. (1992). "Management and theories of organizations in the 1990s: towards a critical radical humanism?". In Academy of Management Review, 17(3), 407-431.

Albatross: an eye on Workplace Learning. (2012). E $\pi i ́ \sigma \eta \mu \eta ~ \imath \sigma \tau о \sigma \varepsilon \lambda i ́ \delta \alpha$. http://www.albatrossjournal.gr Boud, D., Garrick, J. (1999). "Understandings of workplace learning”. $\Sigma$ o o Boud, D. and Garrick, J. (eds.). Understanding Learning at Work. London: Routledge.

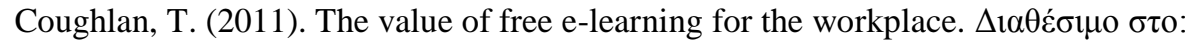

http://www.open.ac.uk/platform/news-and-features/the-value-free-e-learning-the-workplace

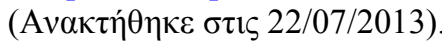

Lee, T., Fuller, A., Ashton, D., Butler, P., Felstead, A., Unwin, L., Walters, S. (2004). Learning as Work: Teaching and Learning Processes in the Contemporary Work Organization. University of Leicester. $\Delta \iota \alpha \theta \dot{\varepsilon} \sigma \mu \mathrm{o} \sigma \tau$ :

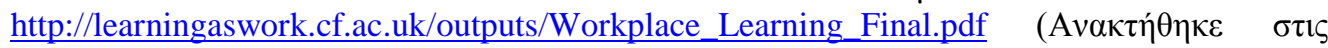
07/08/2013).

Pedler M., Burgoyne J. \& Boydell T. (1997). The Learning Company: A strategy for sustainable development. London: McGraw Hill. 